\title{
Two-year follow-up study of a group-based diabetes medical nutrition therapy and motivational interviewing intervention among African American women
}

\author{
This article was published in the following Dove Press journal: \\ Patient Related Outcome Measures \\ 15 April 2017 \\ Number of times this article has been viewed
}

\author{
Stephania T Miller' \\ Sylvie A Akohoue ${ }^{2}$ \\ 'Department of Surgery, ${ }^{2}$ Department \\ of Family and Community Medicine, \\ Meharry Medical College, Nashville, \\ TN, USA
}

Objectives: To assess the 2-year efficacy of a combined medical nutrition therapy and motivational interviewing (MI) pilot study intervention and factors that influenced long-term dietary self-care.

Research design and methods: Pilot study participants, African American women with type 2 diabetes, completed a 2-year follow-up study visit, including clinical assessments and completion of a dietary self-care questionnaire and a semi-structured interview. Wilcoxon signed-rank tests were used to evaluate differences between baseline and 2-year follow-up clinical and dietary self-care outcomes. Hierarchical coding was used to analyze semi-structured interviews and categorize facilitator and barrier themes into subthemes. Subthemes were quantified based on the number of subtheme-related comments.

Results: Among the 12 participants (mean age 57.1 55.7 years), improvements were observed for $\mathrm{HbA1c}$ (baseline: 10.25\%; interquartile range [IQR]: 8.10, 11.72 and follow-up: 8.8\%; IQR: 7.48,10.22), systolic blood pressure (baseline: $142 \mathrm{~mm} \mathrm{Hg}$; IQR: $134.25,157.25$ and follow-up: $127 \mathrm{~mm} \mathrm{Hg}$; IQR: 113.5, 143.25), frequency of eating high-fat foods (baseline: 3.5 days; IQR: 2.75, 4.25 and follow-up: 3 days; IQR: $2.5,4.5$ ), and of spacing carbohydrates throughout the day (baseline: 3 days; IQR: 3.0, 4.0 and follow-up: 4 days; IQR: 1.5, 4.5). There was a statistically significant decrease $(p=0.04)$ in the frequency of fruit and vegetable intake (baseline: 4 days; IQR: 3.75, 7.0 and follow-up: 3.5 days; IQR: 2.75, 4.0). Dietary self-care barriers and facilitators included internal (eg, motivation) and external factors (eg, social support). Motivation (70 comments) and lack of motivation (67 comments) were the most pervasive facilitator and barrier subthemes, respectively.

Conclusion: Overall, diabetes-related clinical and dietary self-care outcomes were improved following a combined medical nutritional therapy/MI intervention, and motivation played an important role in dietary self-care engagement. Future research is needed to assess the added benefit of MI in improving clinical and dietary self-care outcomes and to identify best strategies to support post-intervention dietary self-care engagement.

Keywords: type 2 diabetes, dietary self-care, motivation interviewing, African American women

\section{Introduction}

African American (AA) women carry disproportionate diabetes-related morbidity burdens, ${ }^{1,2}$ much of which is related to dietary self-care challenges. ${ }^{3}$ The American Diabetes Association recommends that persons with diabetes receive medical nutrition therapy (MNT) to support them in developing dietary self-management plans
Department of Surgery, Meharry Medical College, 1005 Dr DB Todd Jr Boulevard, Nashville, TN 37208, USA

$\mathrm{Tel}+\mathrm{I} 6153275666$

Fax +l 6153275579

Email smiller@mmc.edu 
to improve glycemic, blood pressure, and lipid control and reduce their risk for complications (eg, neuropathy and heart disease) development or progression. ${ }^{4}$ For AA women, incorporating recommended dietary self-care strategies into daily life is challenging due to cultural influences and general day-to-day responsibilities. ${ }^{5}$ In addition, they report low motivation to engage in dietary self-care behaviors. ${ }^{6}$

Given the persistent diabetes burden experienced by AA women and the known benefits of motivational support in improving outcomes for a variety of self-care behaviors, including dietary self-care, ${ }^{7}$ we previously pilot-tested and reported on the acceptability and efficacy of a combined diabetes MNT and motivation-based intervention to improve diabetes and dietary self-care outcomes among AA women. ${ }^{8}$ The study included 24 AA women with type 2 diabetes who participated in certified diabetes educator/dietitian-facilitated sessions, which targeted dietary self-care skills (eg, carbohydrate counting and label reading). Motivational interviewing strategies $^{9}$ were used to encourage increased internal motivation to engage in those skills. Compared to the slope for pre-intervention $\mathrm{HbA} 1 \mathrm{c}$ levels (based on four pre-intervention assessments), there was a significant ( $p=0.03$ ) downward trend shift (improvement) for the slope for post-intervention $\mathrm{HbA} 1 \mathrm{c}$ levels (based on three post-intervention assessments); HbA1c values increasing pre-intervention $(8.8 \pm 2.0$ to $9.7 \pm 2.4)$ and decreasing post-intervention ( $9.4 \pm 1.8$ to $8.8 \pm 2.2$ ). All women reported that they would recommend the study to others. The following verbatim comments reflected common sentiments about study participation: “...The discussions were pivotal in my process. Sometimes, I've felt alone in all this."; and "I enjoyed meeting other women of my ethnic background that have diabetes that struggle to control it."

\section{Materials and methods}

As part of planning and tailoring a future randomized clinical trial based on preliminary intervention efficacy observed in the pilot study, we conducted a follow-up study to assess the 2-year intervention efficacy on diabetes-related clinical and dietary self-care outcomes and factors that influenced dietary self-care during the follow-up period for which there was no study-related contact. Original pilot study participants were invited to a one time, 2-hour follow-up visit at the Meharry Clinical and Translational Research Center. Original pilot study participants met the following study criteria: 1) AA women, 2) $\geq 34$ years of age, 3) clinical type 2 diabetes for at least 6 months, 4) HbA1c $\geq 7.0 \%$, and 5) systolic blood pressure of $\geq 130$, low-density lipoprotein cholesterol of $\geq 100$, or body mass index (BMI) of $\geq 30$. Visits were completed from July 2014 to September 2014. During the first hour, a clinical research nurse assisted in duplicating original pilot study assessments, including blood draws for HbAlc (study outcome), height and weight measurements for BMI (study outcome), and systolic blood pressure (study outcome). Study participants independently completed the validated Summary of Diabetes Self-Care Activities questionnaire, which includes specific dietary self-care assessments (study outcome). ${ }^{10}$ During the second hour, audio-recorded semistructured interviews were conducted by a postdoctoral research associate to identify factors that influenced integration of dietary self-care skills into daily life during the 2-year follow-up period. Participants were asked, "Of the dietary self-management strategies that you engaged in the longest or are still engaged in, what made it easier for you to keep it up? What made it harder?" To support recall, participants were given a list of the dietary self-care skills that were highlighted in the original pilot study, including pictures of self-care tools they received (eg, journals for recording dietary intake). The follow-up study was approved by the Meharry Medical College Institutional Review Board and all participants provided written informed consent.

\section{Data analysis}

To assess the 2-year intervention efficacy on diabetes-related clinical and dietary self-care outcomes, follow-up study measures were compared with those from the original pilot study using Wilcoxon signed-rank tests with a statistical significance level set at 0.05 . Data were analyzed using $\mathrm{R}$ statistical software (version 3.1.2, Vienna, Austria). A hierarchal coding system, method of categorizing qualitative data based on the level of detail or specificity, was used to guide semi-structured interview analysis. The interview questions and a review of one interview transcript were used as the framework for developing the coding system. Dietary self-care facilitators and barriers were categorized as major themes and specific facilitators and barriers as subthemes. Subtheme-related comments were categorized as codes and quantified.

\section{Results}

Follow-up study participants $(n=12)$ were $57.1 \pm 5.7$ years old. More than half $(58.3 \%)$ had attended or graduated from college and most $(91.7 \%)$ were not married. Table 1 compares the baseline and follow-up study outcomes reported as medians. Compared with baseline, improved, yet nonsignificant, changes were observed for $\mathrm{HbA1c}$ (baseline: 10.25\%; interquartile range [IQR]: $8.10,11.72$ and follow-up: 8.8\%; IQR: 7.48, 10.22) and systolic blood 
Table I Clinical and dietary self-care outcomes among African American women enrolled in a combined diabetes medical nutrition therapy and motivational interviewing intervention

\begin{tabular}{|c|c|c|c|}
\hline Study outcomes & Baseline & 2-year follow-up & p-Value \\
\hline HbAlc (\%) & $10.25(8.10,11.72)$ & $8.8(7.48,10.22)$ & 0.204 \\
\hline Systolic blood pressure $(\mathrm{mm} \mathrm{Hg})$ & $142(134.25,157.25)$ & $127(\mid 13.5,143.25)$ & 0.12 \\
\hline BMI $\left(\mathrm{kg} / \mathrm{m}^{2}\right)$ & $40.85(33.65,46.55)$ & $41.1(33.85,43.1)$ & 1.0 \\
\hline Eat 5 fruits/vegetables (days/week) ${ }^{\mathrm{b}}$ & $4.0(3.75,7.0)$ & $3.5(2.75,4.0)$ & 0.04 \\
\hline Eat high-fat foods (days/week) ${ }^{\mathrm{b}}$ & $3.5(2.75,4.25)$ & $3(2.5,4.5)$ & 0.89 \\
\hline $\begin{array}{l}\text { Space carbohydrates evenly } \\
\text { throughout the day (days/week) }\end{array}$ & $3.0(3.0,4.00)$ & $4.0(1.5,4.5)$ & 0.89 \\
\hline
\end{tabular}

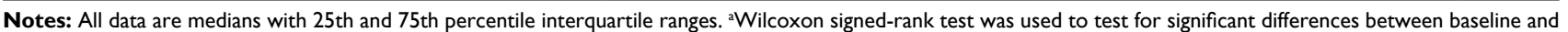
2-year follow-up measures. ${ }^{b}$ From summary of diabetes self-care activities questionnaire; ${ }^{10}$ score range $0-7$ days/week.

Abbreviation: BMI, body mass index.

pressure (baseline: $142 \mathrm{~mm} \mathrm{Hg}$; IQR: $134.25,157.25$ and follow-up: $127 \mathrm{~mm} \mathrm{Hg}$; IQR: 113.5, 143.25). There was no significant change in BMI (baseline: $40.85 \mathrm{~kg} / \mathrm{m}^{2}$; IQR: $33.65,46.55$ and follow-up: $41.1 \mathrm{~kg} / \mathrm{m}^{2}$; IQR: $33.85,43.1$ ). Frequency of eating high-fat foods decreased (baseline: 3.5 days; IQR: 2.75, 4.25 and follow-up: 3 days; IQR: 2.5, 4.5) and of spacing carbohydrates throughout the day increased (baseline: 3 days; IQR: 3.0, 4.0 and follow-up: 4 days; IQR: $1.5,4.5)$, although changes were not statistically significant. There was a statistically significant $(p=0.04)$ decrease in the frequency of fruit and vegetable intake (baseline: 4 days; IQR: 3.75, 7.0 and follow-up: 3.5 days; IQR: $2.75,4.0$ ). Relative to factors influencing engagement in specific dietary self-care skills, based on semi-structured interview analysis (Table 2), barrier-related subthemes $(\mathrm{n}=10)$ exceeded facilitator themes $(\mathrm{n}=7)$ with both categories including internal (eg, motivation)

Table 2 Dietary self-care facilitators and barriers among African American women with type 2 diabetes $(n=12)$

\begin{tabular}{ll}
\hline $\begin{array}{l}\text { Emerging semi-structured } \\
\text { interview themes }\end{array}$ & $\begin{array}{l}\text { Theme-related } \\
\text { Codes }\end{array}$ \\
\hline Facilitators & N \\
\hline Motivation & 70 \\
Knowledge gained during intervention & 57 \\
Anticipated health benefits & 51 \\
Positive peer support & 50 \\
Positive family support & 39 \\
Positive health care professional support & 28 \\
Convenience & 25 \\
Barriers & \\
Lack of motivation & 67 \\
Inconvenience & 54 \\
Competing priorities & 49 \\
Finances & 44 \\
Problems remembering & 39 \\
Lack of knowledge & 30 \\
Lack of family support & 24 \\
Stress & 15 \\
Lack of confidence & 13 \\
Lack of peer support & 7 \\
\hline
\end{tabular}

and external factors (eg, peer support, finances, and competing priorities). Half of the barrier subthemes were articulated as the absence of facilitators (eg, social support viewed as a facilitator but lack thereof as a barrier). The most codes, or subtheme-related comments, were assigned to the motivation facilitator subtheme $(\mathrm{n}=70)$ and the lack of motivation barrier subtheme ( $\mathrm{n}=67)$.

\section{Discussion}

We assessed the 2-year efficacy of a combined diabetes MNT and motivation-based intervention on diabetes-related clinical and dietary self-care outcomes among AA women. Although there were improvements in key outcomes, such as glycemic control, as measured by $\mathrm{HbA} 1 \mathrm{c}$, and systolic blood pressure, the changes were not significant. As we did observe a statistically significant trend for improvement in glycemic control when comparing pre- and post-intervention values and immediate post-intervention values in the pilot study, ${ }^{8}$ the results here suggest that those early improvements trends did not continue. It is encouraging, however, that, with the exception of fruit and vegetable intake, clinical and dietary self-care outcome trends were improved overall. These improvement trends are consistent with previous research noting the long-term benefits of lifestyle interventions on outcomes. ${ }^{11}$

Our qualitative assessment of factors that influenced dietary self-care during the 2-year follow-up period revealed barriers to dietary self-care that are consistent with previous research among both rural and urban AA women with type 2 diabetes. For example, there is ample evidence that factors such as competing priorities, financial burden of self-care, and limited social support negatively influence women's attempts to initiate and maintain recommended dietary self-care behaviors. ${ }^{6,12}$ It was not surprising that some barriers were viewed as the absence of a facilitator. For example, lack of family support was a perceived barrier, so it follows that having 
positive family support would be viewed as facilitator of engagement in dietary self-care management. It is noteworthy, however, that motivation and the lack thereof, based on code counts, emerged as the most pervasive facilitator and barrier subthemes, respectively. A validated theoretical framework emphasizing the critical role of motivation in diabetes selfcare engagement, in addition to receiving accurate information and being taught behavioral skills, ${ }^{13}$ support this finding. These observations are also consistent with previous work documenting low motivation for diabetes self-care among AA women. ${ }^{6}$ This follow-up study is the first to document the relevance of motivation to long-term diabetes-related dietary self-care efforts among a patient group at high risk for poor diabetes outcomes. This is particularly salient given the need to identify factors to prevent self-care relapse following diabetes self-care interventions. ${ }^{14}$

Although the sample size was restricted to participants enrolled in the original pilot study, quantitative data analysis and interpretation were further limited because all participants did not complete the follow-up study. In particular, definitive conclusions about the 2-year efficacy of the original pilot study on the reported outcomes, such as HbAlc, are not possible. In addition, information that could have influenced 2-year outcomes, such as medication adjustment, was not collected. While also a limitation for the qualitative follow-up study component, the sample size did not prevent the emergence of common dietary self-care facilitators and barriers among participants who completed the visit. Their shared perceptions regarding barriers and facilitators to longterm dietary self-care management, including the critical role of motivation that is also consistent with existing research, support the validity of the qualitative data findings and their value in optimizing future studies.

\section{Conclusion}

Diabetes MNT is an evidence-based strategy for reducing the risk of diabetes-related complications for which AA women carry a disproportionate burden. ${ }^{1,2}$ Motivational interviewing, in a variety of contexts, has helped patients improve their health outcomes. ${ }^{15}$ This study indicates that combining MNT and motivational interviewing may be an effective approach for promoting dietary self-care and improving clinical outcomes among AA women. Future research, in a larger sample size, should assess whether the addition of the motivational interviewing component results in greater improvement in diabetes and dietary self-care outcomes compared with MNT alone. Additional research is also needed to identify best strategies to support post-intervention dietary self-care engagement. This additional research will inform ongoing efforts to tailor MNT in a way that those at highest risk receive optimal benefit.

\section{Acknowledgments}

This work was supported by National Institutes of Health grants R34DK090670, UL1TR000445, and U54MD007593. The authors wish to thank the study participants for their time, dedication, and selflessness in sharing their diabetesrelated struggles and successes during the study follow-up period and attending a group meeting to hear and provide comments about the results.

\section{Disclosure}

The authors report no conflicts of interest in this work.

\section{References}

1. Centers for Disease Control and Prevention. Age-adjusted incidence of end-stage renal disease related to diabetes mellitus (ESRD-DM) per 100,000 diabetic population, by race, ethnicity, and sex, United States, 1980-2008; 2013. Available from: http://www.cdc.gov/diabetes/ statistics/esrd/fig5.htm. Accessed April 1, 2016.

2. Centers for Disease Control and Prevention. National Vital Statistics Report; 2014. Available from: http://www.cdc.gov/nchs/data/nvsr/ nvsr60/nvsr60_03.pdf. Accessed April 1, 2016.

3. Amend A, Melkus GD, Chyun DA, Galasso P, Wylie-Rosett J. Validation of dietary intake data in black women with type 2 diabetes. $J$ Am Diet Assoc. 2007;107(1):112-117.

4. Bantle JP, Wylie-Rosett J, Albright AL, et al. Nutrition recommendations and interventions for diabetes: a position statement of the American Diabetes Association. Diabetes Care. 2008;31(Suppl 1):S61-S78.

5. Samuel-Hodge CD, Headen SW, Skelly AH, et al. Influences on dayto-day self-management of type 2 diabetes among African-American women: spirituality, the multi-caregiver role, and other social context factors. Diabetes Care. 2000;23(7):928-933.

6. Miller ST. Diabetes and psychological profile of younger rural African American women with type 2 diabetes. $J$ Health Care Poor Underserved. 2011;22(4):1239-1252.

7. Emmons KM, Rollnick S. Motivational interviewing in health care settings. Opportunities and limitations. Am J Prev Med. 2001;20(1):68-74.

8. Miller ST, Oates VJ, Brooks MA, Shintani A, Gebretsadik T, Jenkins DM. Preliminary efficacy of group medical nutrition therapy and motivational interviewing among obese African American women with type 2 diabetes: a pilot study. J Obes. 2014:345941.

9. Miller W, Rollnick S. Motivational Interviewing: Preparing People for Change. 2nd ed. New York, NY: The Guilford Press; 2000.

10. Toobert DJ, Hampson SE, Glasgow RE. The summary of diabetes self-care activities measure: results from 7 studies and a revised scale. Diabetes Care. 2000;23(7):943-950.

11. Nicoll KG, Ramser KL, Campbell JD, et al. Sustainability of improved glycemic control after diabetes self-management education. Diabetes Spectr. 2014;27(3):207-211.

12. Chlebowy DO, Hood S, LaJoie AS. Gender differences in diabetes selfmanagement among African American adults. West J Nurs Res. 2013; 35(6):703-721.

13. Osborn CY, Egede LE. Validation of an information-motivationbehavioral skills model of diabetes self-care (IMB-DSC). Patient Educ Couns. 2010;79(1):49-54.

14. Huizinga MM, Gebretsadik T, Garcia Ulen C, et al. Preventing glycaemic relapse in recently controlled type 2 diabetes patients: a randomised controlled trial. Diabetologia. 2010;53(5):832-839.

15. Ekong G, Kavookjian J. Motivational interviewing and outcomes in adults with type 2 diabetes: a systematic review. Patient Educ Couns. 2016;99(6):944-952. 


\section{Publish your work in this journal}

Patient Related Outcome Measures is an international, peer-reviewed, open access journal focusing on treatment outcomes specifically relevant to patients. All aspects of patient care are addressed within the journal and practitioners from all disciplines are invited to submit their work as well as healthcare researchers and patient support groups.
The journal is included in PubMed. The manuscript management system is completely online and includes a very quick and fair peer-review system. Visit http://www.dovepress.com/testimonials.php to read real quotes from published authors.

Submit your manuscript here: http://www.dovepress.com/patient-related-outcome-measures-journal 